\title{
Large Eddy Simulations on a pitching airfoil: Analysis of the reduced frequency influence
}

\author{
N Guillaud ${ }^{1}$, G Balarac ${ }^{2}$ and E Goncalvès ${ }^{3}$ \\ ${ }^{1}$ HydroQuest SAS, INOVALLIA, bâtiment B, 16 chemin de Malacher, 38240 Meylan, France \\ 2 Univ. Grenoble Alpes / CNRS, LEGI UMR 5519, Grenoble, F-38041, France \\ 3 ISAE-ENSMA/CNRS, Pprime UPR 3346, Téléport 2, 1 Avenue \\ Clément Ader, BP 40109, 86961 Futuroscope Chasseneuil Cedex, France
}

(Dated: June 7, 2017)

\begin{abstract}
Large Eddy Simulations (LES) have been performed on a pitching NACA0012 airfoil at $R e_{c}=2.10^{4}$. The influence of the reduced frequency on the dynamic stall phenomenon is investigated. Special attention is given on the aerodynamic coefficients drop incidence delay in comparison with a static case. The analysis is based on vorticity field observations and the introduction of local lift and friction coefficients. The boundary layer events and the presence and the impact of a Leading-Edge Vortex (LEV) are investigated. The total stall delay is decomposed into two main phases: a delay in the boundary layer separation which occurs at higher incidence as the reduced frequency increases and the initiation and the growth of the LEV. For selected reduced frequencies, the influence of the LEV on the lift and drag coefficients is analysed as a function of the airfoil angle of incidence and then as a function of time. The LEV maintains a high level of lift and drag. Its lifetime on the airfoil suction side seems to be shortened by the beginning of the airfoil downstroke motion. Given the fact that the LEV appears at higher incidence as the reduced frequency grows, the LEV lifetime on the airfoil suction side decreases as the reduced frequency increases. However, the airfoil oscillation being faster, the LEV goes through larger incidence ranges during its lifetime and leads to a larger lift and drag drop incidence delay as the reduced frequency increases.
\end{abstract}




\section{INTRODUCTION}

The dynamic stall phenomenon appears when flow incidence on an airfoil is time dependent. It is thus a widespread issue in industry and in nature. The blade motion of an helicopter or that of a vertical axis wind turbine are two famous fields of application of the dynamic stall phenomenon studies. More recently, studies at lower Reynolds number aim to better understand the dynamics of insect flight for bioinspired micro aerial vehicles issues. Due to its large field of application, its impact on technology performances and its complexity, the dynamic stall phenomenon has been studied for decades. A common case used to study the dynamic stall phenomenon is an airfoil with a pitching motion. Numerous experimental studies such as [1] showed that a pitching airfoil could stalls at higher angle of incidence than a static airfoil leading to higher maximum values of lift and drag coefficients. However, the reattachment of the boundary layer is delayed at lower angle of incidence than in the static airfoil configuration, leading to a hysteresis loop in the aerodynamic coefficients evolution. According to [2], the dynamic stall term is used to describe the series of events which lead to the delay of an airfoil stall. When static angle of incidence is exceeded, Corke and Thomas [3] reported two major mechanisms responsible for the stall delay: a delay in the boundary layer separation and the initiation and growth of a Leading Edge Vortex (LEV). The LEV has been investigated in many experimental studies. Mechanisms of initiation, growth and detachment of the LEV has been studied by [4-7] but are still challenging to understand as witness recent studies [8,9]. A deeper understanding of the dynamic stall phenomenon is of primary interest to improve semi-empirical models like Leishman and Beddoes model [10] which have been proposed to predict the aerodynamic coefficients of an airfoil undergoing dynamic stall. New control techniques of the dynamic stall phenomenon could also emerge from a better dynamic stall phenomenon comprehension.

Previous numerical studies showed that dynamic stall phenomenon is challenging to predict. Two-dimensional Unsteady Reynolds Averaged Navier-Stokes (URANS) approaches have been tested for example by Wang et al. [11]. They reproduced the experimental NACA0012 pitching airfoil cases studied by Wernert et al. [12] and Lee and Gerontakos [5]. Both $k-\omega$ and $k-\omega$ SST turbulence models have been evaluated on their ability to correctly reproduce the velocity profiles along the airfoil suction side, the global flow topology and the lift and drag coefficient evolution. The $k-\omega$ SST turbulence model gave better results, but both failed to correctly predict dynamic stall phenomenon. The stall delay could be underestimated and the aerodynamic coefficient loop is not well reproduced. Two-dimensional URANS approaches also have been tested by Martinat et 
al. [13] and compared to the experiments of Berton et al. [14] and McAlister et al. [15]. Authors' conclusions are comparable to those of Wang et al.. Due to the inaccuracy of URANS approaches in configurations with deep dynamic stall, hybrid approaches, which use URANS approach in the near-wall region and LES away from it, have been evaluated for example by Martinat et al. [13] and Sanchez-Rocha et al. [16]. Martinat et al. evaluated a DDES [17] $k-\omega$ SST three-dimensional hybrid approach. In comparison with two-dimensional URANS approaches, the hybrid approach seems to improve the flow topology prediction in the downstroke part of the airfoil movement. Nevertheless, the prediction of the aerodynamic coefficients is still not satisfying. Sanchez-Rocha et al. [16] described and used another hybrid approach based on $k-\omega$ SST URANS model and a localized dynamic $k^{s g s}$ one-equation LES model. The comparison between two-dimensional and three-dimensional results confirmed the need to perform three-dimensional computations to improve the flow prediction in a deep dynamic stall configuration. The aerodynamic coefficients obtained with the three-dimensional computations are closed to the experimental results taken from [18] but the lift coefficient is overestimated in the downstroke part of the pitching movement. Due to the expensive computational cost of such simulations, the authors could not confirm if this discrepancy originated from the wingspan of the computational domain, a lack of convergence of statistics or from the proposed hybrid approach. A LES approach on a piching airfoil have been used by Rahromostaqim et al. [19]. The airfoil is represented with an immersed boundary method [20]. The computation validation is based on the variation amplitude of the lift coefficient compared to its averaged value. The discrepancy between the numerical result and the experimental result taken from [21] is low since the computation overestimated this quantity about only 3.5\%. Garmann and Visbal [22] used an implicit LES approach to study an oscillating flat plate. The obtained lift and drag coefficients are in close agreement with the experimental results, despite small discrepancies during the downstroke part of the movement. According to the previous mention studies, LES approach seems to be appropriate to correctly reproduced the dynamic stall phenomenon. However, probably due to their computational cost, studies on pitching airfoils with a LES approach are today rare.

The present study proposes a numerical investigation of the dynamic stall phenomenon with a LES approach. Among all free parameters in a pitching airfoil configuration, several experimental studies $[1,23,24]$ showed that the reduced frequency $k=\pi f c / V_{0}$ ( $f$ being the oscillation frequency, $c$ the airfoil chord length and $V_{0}$ the freestream velocity) has a strong influence on the dynamic stall phenomenon and is thus a key parameter. This study aims to better understand the influence 
of the reduced frequency on the dynamic stall phenomenon with a special attention given on the aerodynamic coefficients drop incidence delay in comparison with a static case. The analysis will be based on vorticity field observations and the introduction of local lift and friction coefficients. The boundary layer events and the presence and the impact of a Leading-Edge Vortex (LEV) will be investigated. For selected reduced frequencies, the influence of the LEV on the aerodynamic coefficients will be analysed as a function of incidence and then as a function of time.

\section{STUDY CASES AND NUMERICAL SET UP}

Figure 1 illustrates the studied pitching airfoil configuration. It is a NACA0012 airfoil with a pitch axis located at 1/4-chord from the leading edge. The flow incidence on the airfoil varies as:

$$
\alpha(t)=\alpha_{m}+\alpha_{a} \sin (2 \pi f t)
$$

where $\alpha_{m}=10^{\circ}$ is the mean incidence angle, $\alpha_{a}=10^{\circ}$ the amplitude, $f$ the airfoil oscillation frequency and $t$ the time. With the freestream velocity $V_{0}$, the airfoil chord length $c$ and the

kinematic viscosity $\nu$, the Reynolds number based on the airfoil chord length $R e_{c}=\frac{V_{0} c}{\nu}$ is set to $2 \times 10^{4}$. The flow is assumed to be incompressible. Four reduced frequencies $k=\pi f c / V_{0}$ are studied. These reduced frequencies are listed in table I which also recalls the principal parameters of the study cases. Computations on a static airfoil at various incidences also have been carried out to be compared to dynamic cases.

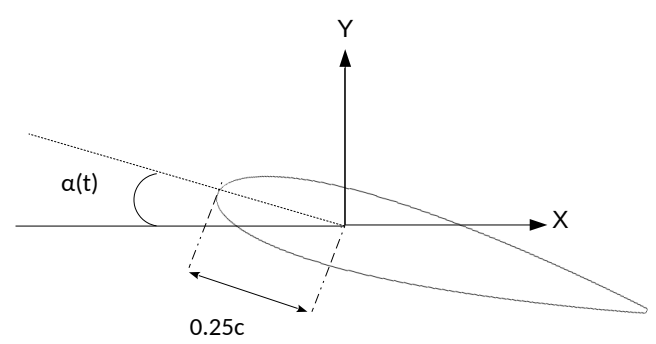

FIG. 1: Studied pitching airfoil.

\begin{tabular}{cc}
\hline \hline Profile & NACA 0012 \\
$R e_{c}$ & $2 \times 10^{4}$ \\
$\alpha_{m}$ & $10^{\circ}$ \\
$\alpha_{a}$ & $10^{\circ}$ \\
$k$ & $0.025 ; 0.1 ; 0.2 ; 0.4$ \\
\hline \hline
\end{tabular}

TABLE I: Pitching airfoil parameters.

Computations were performed using the YALES2 flow solver [25]. This code solves the incompressible and low-Mach number Navier-Stokes equations for turbulent flows on unstructured 
meshes using a projection method for pressure-velocity coupling [26]. It relies on fourth-order central finite-volume schemes and on highly efficient linear solvers [27], which enable the simulation and the post-processing of iso-thermal, reacting or multiphase flows on massive unstructured grids [28-30]. The time integration is explicit for convective terms with a fourth-order scheme. It is a modification of the classic RK4 scheme called TFV4A [31]. To avoid a too restrictive condition on the time step, computation of diffusive terms is implicit. A constant Courant-Friedrichs-Lewy (CFL) number of 0.9 is set for all cases. The Smagorinsky dynamic subgrid-scale model is used [32]. To avoid rotor/stator interface, equations are expressed in a rotating frame and a rotating velocity condition is imposed at the inlet. The domain extension is equal to 30c around the airfoil and $0.5 \mathrm{c}$ in the spanwise direction where periodic boundary conditions are imposed. A wall condition is imposed on the airfoil surface. No wall laws are used. As no turbulence is imposed at the inlet, the flow is laminar upstream from the airfoil. Computation domain and boundary conditions are illustrated on figure 2.

The mesh is generated with ANSYS Meshing. It is composed of prisms at wall and tetrahedron elsewhere. It contains approximately 5 million cells. The most likely value of first cell size equals to 0.7 wall unit along the airfoil and is always smaller than 5.6 wall unit. The aspect ratio of the prisms at the walls is approximately equal to 8. The element growth rate from the wall is equals to 1.1. The mesh is illustrated on figure 3 .

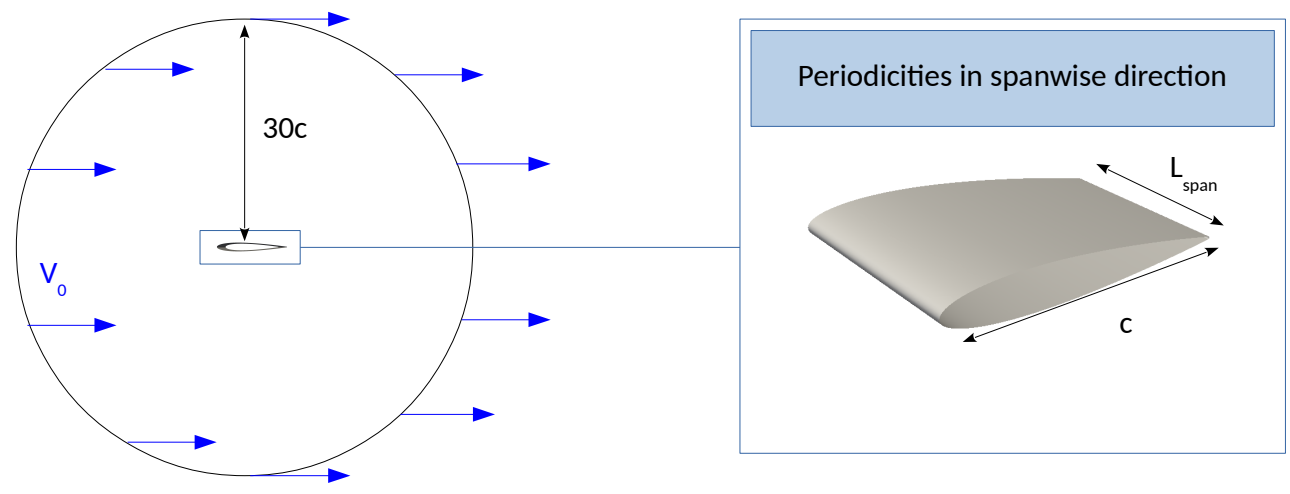

FIG. 2: Computation domain illustration.

\section{ANALYSIS METHODOLOGY}

To investigate the aerodynamic coefficients drop incidence delay for pitching airfoil, phaseaveraged lift and drag coefficient (noted $C_{l}$ and $C_{d}$, respectively) loops will be shown for each 


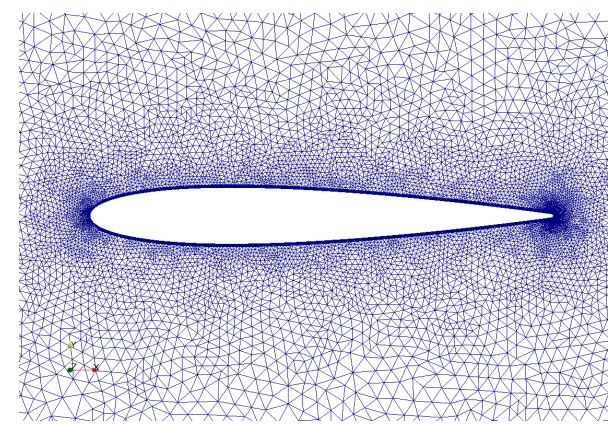

(a)

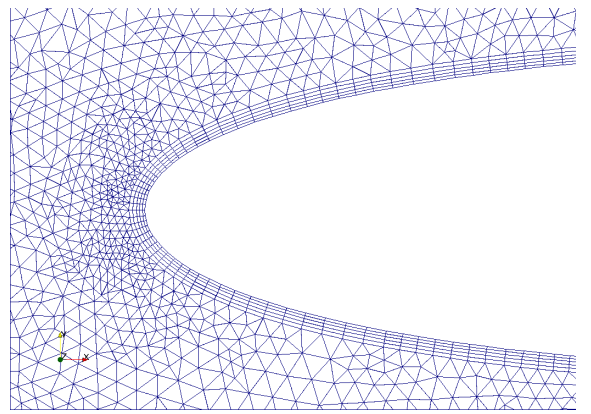

(b)

FIG. 3: Illustration of the mesh - 3a Mesh around the airfoil, 3b Enlargement of the mesh around the leading edge region.

reduced frequency.

To perform a deeper analysis, the airfoil suction side is divided into 10 stripes on which forces are integrated at each time step to obtain a local lift coefficient $C_{l}$ local:

$$
C_{\text {local }}(x / c, \alpha)=\frac{1}{0.5 \rho S_{\text {stripe }} V_{0}^{2}} \iint_{S_{\text {stripe }}}\left(-P \vec{n}_{I}+\overline{\bar{\tau}} \cdot \vec{n}_{I}\right) \cdot \vec{e}_{y} d S
$$

with $\rho$ the fluid density, $P$ the pressure, $\overline{\bar{\tau}}$ the viscous stress tensor, $\vec{n}_{I}$ the unit surface normal at the point $I$ of the airfoil suction side, $\vec{e}_{y}$ the unit vector in the $Y$ direction, $S_{\text {stripe }}$ the area of a stripe and $d S$ the stripe area element. An illustration of a stripe used for the computation of $C_{l \text { local }}$ is depicted on figure 4 . The local lift coefficient is therefore an instantaneous quantity and depends on the position along the airfoil suction side. In the following, the local lift coefficient will be plotted as a function of the incidence and the dimensionless position along the airfoil suction side $x / c$. This quantity will also be plotted as a function of time and $x / c$.

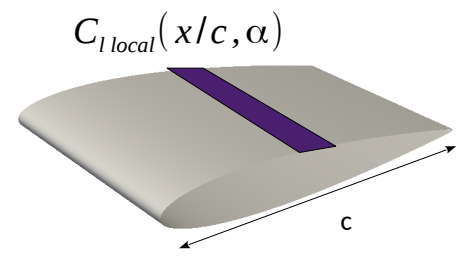

FIG. 4: Illustration of the surfaces used for local lift coefficient computation.

To monitor the evolution of the boundary layer events over a pitching cycle, a local friction 
coefficient is computed on the previous mentioned stripes:

$$
C_{f \text { local }}(x / c, \alpha)=\frac{1}{0.5 \rho S_{\text {stripe }} V_{0}^{2}} \iint_{S_{\text {stripe }}} \overline{\bar{\tau}} \cdot \vec{n}_{I} \cdot \overrightarrow{\tan }_{I} d S
$$

where $\overrightarrow{\tan }_{I}$ is a vector tangent to the airfoil suction side at the point $I$ and located in a streamwise plane. The boundary layer detachment point is detected by a change of sign of $C_{f}$ local . Once the boundary layer detachment point reached the leading edge region, $C_{f}$ local is used to follow the initiation and the evolution of the LEV, since this vortex generates a strong negative friction on the airfoil suction side.

The analysis will be completed with visualizations of instantaneous spanwise vorticity fields in the airfoil midplane. A global view of the three-dimensional flow around the airfoil will be provided by Q-criterion [33] isosurfaces which will highlight the principal vortex of the flow.

The results of the analysis methodology described here are presented and commented in the next section.

\section{RESULTS AND DISCUSSION}

\section{A. Results overview}

Figure 5 shows a Q-criterion isosurface around the airfoil at the incidence $\alpha=15^{\circ}$ during the upstroke phase. In addition to the pitching cases, the result for a static airfoil is depicted. Despite the same incidence, many differences are observed between the different cases. In the static case and the oscillating case at $k=0.025$, the flow is stalled and important vortices are shed into the wake. For the pitching case with $k=0.1$, the flow is not entirely detached from the airfoil. Vorticies stay close to the airfoil suction side. In the oscillating case at $k=0.2$, the generation of a LEV from the leading edge is visible. Few vortices are observed downstream the LEV. In the pitching case with $k=0.4$, the LEV is still not visible. Small two-dimensional vortices are shed from the leading edge vicinity.

These discrepancies lead to different values of lift and drag coefficients. Phase-averaged lift and drag coefficients are depicted as a function of the airfoil angle of incidence on figure 6 . The airfoil upstroke phases are plotted in plain lines and airfoil downstroke phases are plotted in dashed lines. Static lift and drag coefficients have also been reported on this figure.

In accordance with previous experimental observations, e.g. [1], the airfoil motion leads to delay the lift coefficient fall towards higher incidences. The higher the reduced frequency, the higher the 


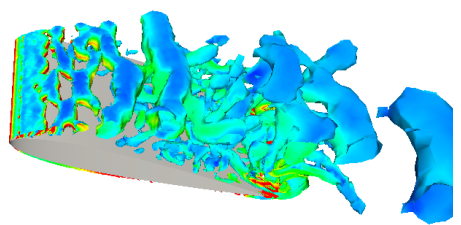

(a) Static

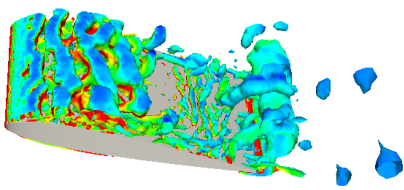

(d) Pitching, $k=0.2$

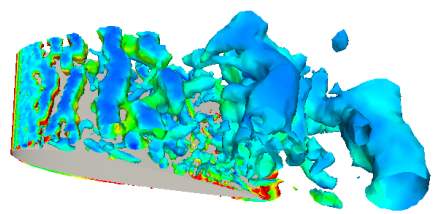

(b) Pitching, $k=0.025$

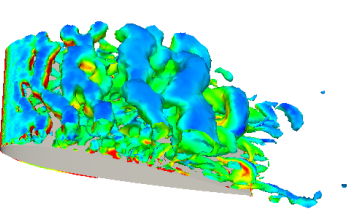

(c) Pitching, $k=0.1$

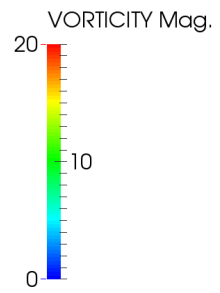

(e) Pitching, $k=0.4$

FIG. 5: Q-criterion isosurface colored by non-dimensional vorticity magnitude, $\omega c / V_{0}$, on the airfoil suction side at an incidence $\alpha \approx 15^{\circ}$. Static and pitching cases, upstroke part of the motion for pitching cases.

incidence delay. The lift coefficient falls around $\alpha=12^{\circ}$ in the pitching case with $k=0.025$ whereas it plunges at the end of the airfoil upstroke when $k=0.2$. With the highest reduced frequency $k=0.4$, it occurs during the airfoil downstroke. The lift coefficient reaches higher values in pitching cases than in a static case. However, once the flow is stalled, the lift coefficient level is similar in all cases. The lift loss at stall in the oscillating cases is therefore higher than in the static case. Finally, an important hysteresis phenomenon is observed in the pitching cases. Lift values observed during upstroke phases are higher than those observed in the static case but become lower in downstroke phases.

Drag coefficient evolutions are similar until a critical incidence for which the drag coefficient of the pitching cases increases in a more or less significant manner compared to the static case. This critical incidence depends on the reduced frequency. The higher the reduced frequency value, the higher this critical incidence. The drag coefficient then falls more or less drastically and reaches values similar to those of the static case. Similarly the lift coefficient, the drag coefficient evolution in the pitching cases shows a hysteresis phenomenon.

To link the evolution of the phase-averaged lift and drag coefficient with the flow around the airfoil, fields of non-dimensional spanwise vorticity are shown on figures 7 to 14 . 


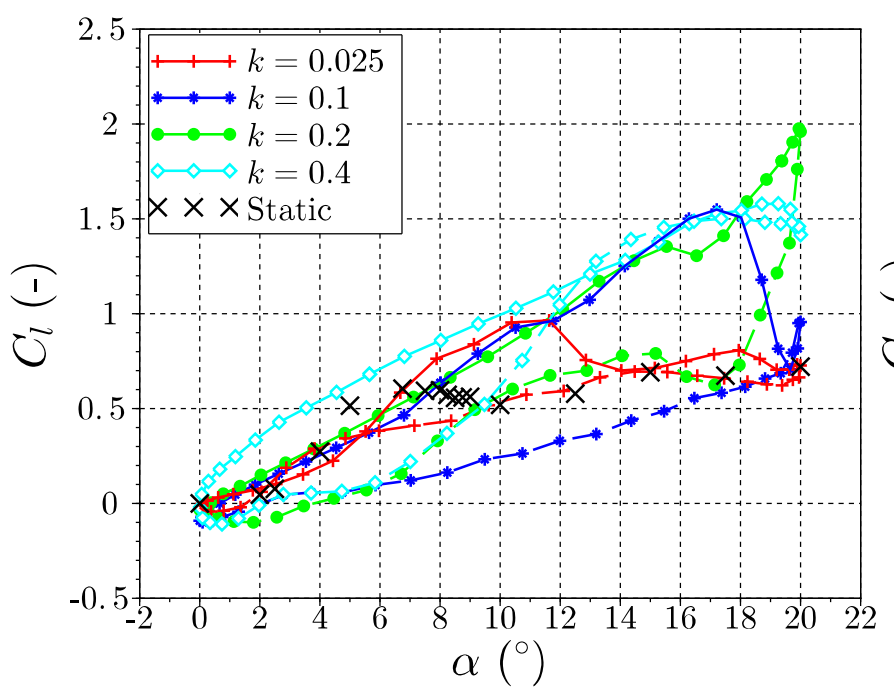

(a)

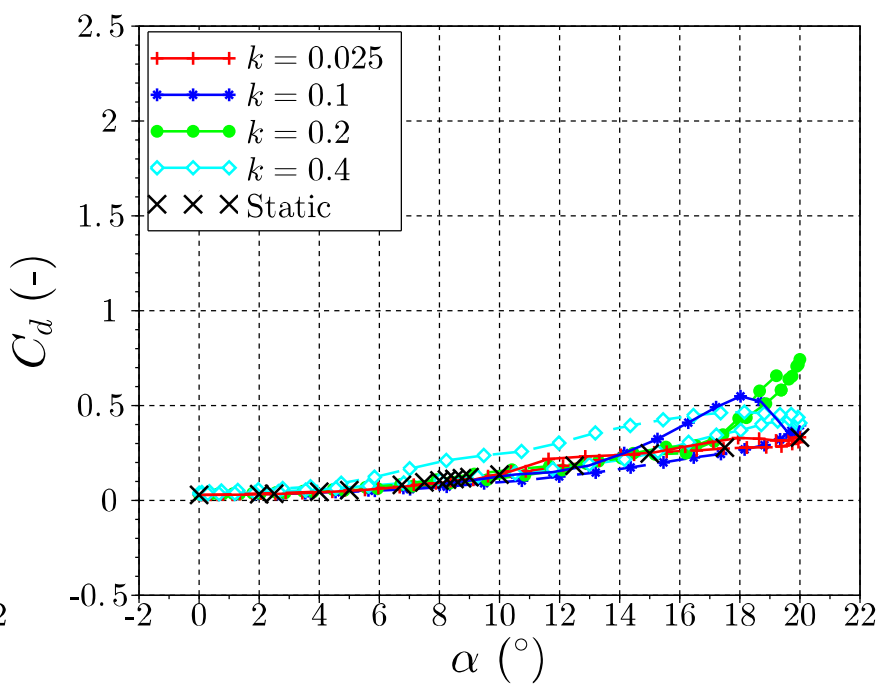

(b)

FIG. 6: Phase-averaged lift coefficient (a) and phase-averaged drag coefficient (b) as a function of the airfoil angle of incidence for different reduced frequencies. Airfoil upstroke phases are depicted in plain lines and airfoil downstroke phases are depicted in dashed lines.

Figure 7 shows the fields at an incidence equal to $6.2^{\circ}$, in upstroke phase for pitching cases. In the static case, the boundary layer is already detached from the trailing edge over an important part of the airfoil suction side. The extent of the boundary layer detachment in the pitching case with $k=0.025$ seems to be lower than in the static case but higher than in the pitching cases with higher reduced frequencies. Pitching cases with $k=0.1, k=0.2$ and $k=0.4$ show similar flows around the airfoil. In all cases, the flow is attached around the leading edge and the airfoil incidence leads to generate a lift force. The drag force is similar in all cases.

When incidence increases to $10^{\circ}$, figure 8 shows that the flow around the static airfoil is stalled. The lift coefficient decreased compared to the maximum level it reached for inferior incidences. In the pitching case with $k=0.025$, the flow is not fully stalled and lift and drag coefficients still increase when incidence grows. For higher reduced frequencies, lift and drag coefficients also increase when incidence grows but the boundary layer detachment process is less and less advanced as the reduced frequency increases.

When the angle of incidence increases to $14.5^{\circ}$, figure 9 shows that the flow in the pitching case with $k=0.025$ is now stalled as the static case. The airfoil lift coefficient felt and reached a level similar to that of the static case. In the pitching case with $k=0.1$, a LEV is clearly visible. A 
LEV is also observed in the pitching case with $k=0.2$ but it is less developed at this incidence. It covers a smaller part of the airfoil suction side. In the pitching case with $k=0.4$, the very beginning of the LEV creation is observed at the leading edge vicinity. The lift coefficient still increases as the incidence grows for pitching cases with $k=0.1, k=0.2$ and $k=0.4$. For $k=0.1$, the drag coefficient slope is henceforth higher.

When incidence increases to $15.9^{\circ}$, figure 10 shows that the LEV generated in the pitching cases with $k=0.1, k=0.2$ and $k=0.4$ develops and extends on a growing area of the airfoil suction side. The higher the reduced frequency, the less developed is the LEV. Lift and drag coefficients continue to increase with incidence. For $k=0.2$, the drag and the lift coefficient slopes become higher around this angle of incidence.

We can observe on figure 11 that when the angle of incidence increases to $19.5^{\circ}$, the flow around the pitching airfoil with $k=0.1$ is now stalled from the suction side, the LEV has been shed into the wake. Lift and drag coefficients felt. A counter-rotating Trailing Edge Vortex (TEV) is clearly visible. For pitching cases with $k=0.2$ and $k=0.4$, LEV still grows along the airfoil suction side. The LEV development in the case $k=0.2$ is still more advanced than in the case $k=0.4$ and covers a larger part of the airfoil. Lift and drag coefficients are closed to their maximum value. The deceleration of the airfoil close to the maximum angle of incidence seems to cause a slight temporary decrease of the lift and drag coefficients for the case with $k=0.4$. Reached values with $k=0.2$ are higher than those with $k=0.4$.

The airfoil then starts its downstroke phase. Figure 12 shows that at an angle of incidence equal to $18.1^{\circ}$ the flow is now stalled from the pitching airfoil at $k=0.2$, the LEV has been shed into the wake. As previously for $k=0.1$ a TEV is also developing. Lift and drag coefficients felt. For $k=0.4$, the LEV is still on the airfoil suction side and continues to grow. Lift coefficient value remains high and drag coefficient reaches its maximum value.

When the airfoil incidence decreases to $11.6^{\circ}$, we see on figure 13 that for the case with $k=0.4$ the LEV passes over the airfoil suction side and will be shed into the wake. Lift and drag coefficients decrease but in a smoother manner compared to the other pitching cases.

Finally, when incidence continues to decrease, the flow over the airfoil starts to reattach from the leading edge to the trailing edge. Figure 14 shows that the progress of the boundary layer reattachment at a given incidence depends on the reduced frequency. The higher the reduced frequency, the more delayed is the process.

Lift and drag coefficients gradually recover the values reached during the upstroke phase, al- 
though it only happens at the beginning of a new cycle for the lift coefficient of the pitching cases with a reduced frequency greater than 0.1 .

According to the present description and in accordance with [3] the incidence delay of the lift and drag coefficients drop in a pitching airfoil configuration is due to two successive phenomena: a boundary layer detachment which happens at higher incidences and the initiation and growth of a LEV after the boundary layer detachment. The rest of the paper is dedicated to the analysis of these two phenomena.

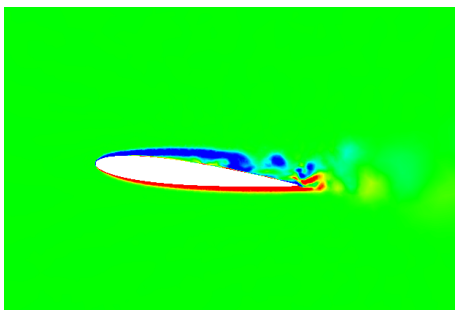

(a) Static

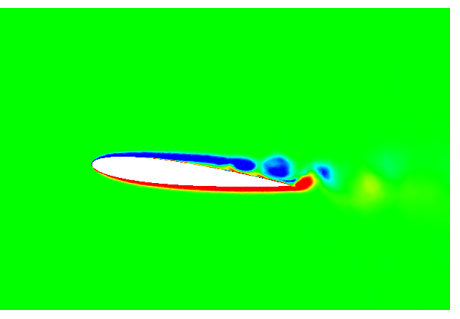

(b) Pitching, $k=0.025$

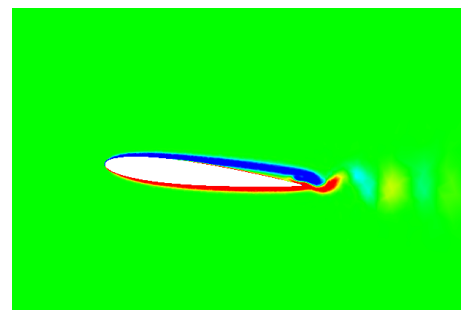

(c) Pitching, $k=0.1$

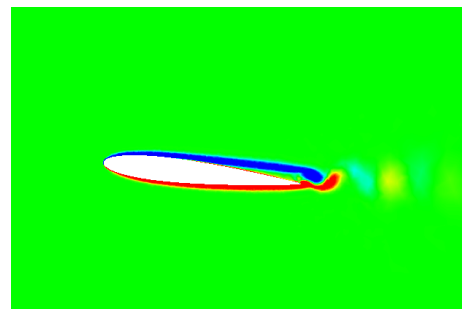

(d) Pitching, $k=0.2$

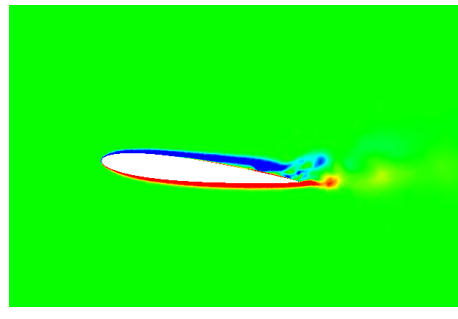

(e) Pitching, $k=0.4$

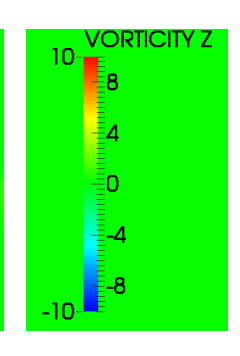

FIG. 7: Contours of non-dimensional spanwise vorticity, $\omega_{Z} c / V_{0}$, at airfoil midplane for a static airfoil and for airfoils pitching at various reduced frequencies. The angle of incidence is $\alpha=6.2^{\circ}$ (upstroke phase). 


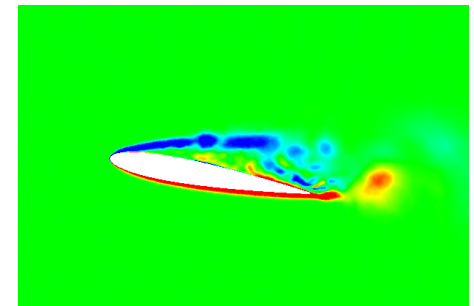

(a) Static

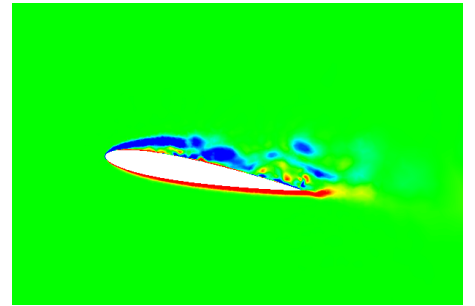

(b) Pitching, $k=0.025$

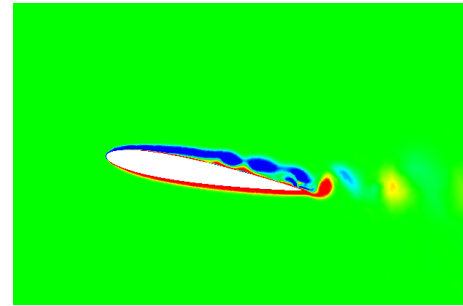

(c) Pitching, $k=0.1$

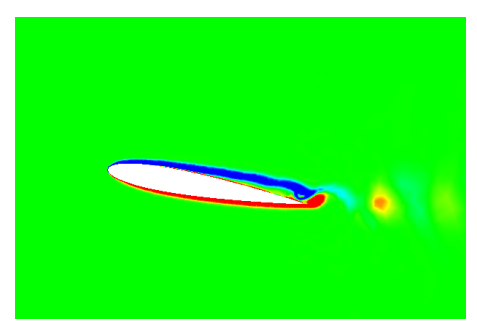

(d) Pitching, $k=0.2$

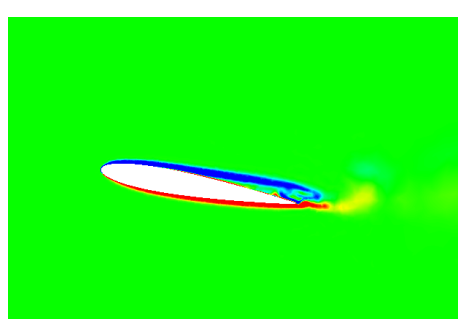

(e) Pitching, $k=0.4$

FIG. 8: Contours of non-dimensional spanwise vorticity, $\omega_{Z} c / V_{0}$, at airfoil midplane for a static airfoil and for airfoils pitching at various reduced frequencies. The angle of incidence is $\alpha=10^{\circ}$ (upstroke phase).

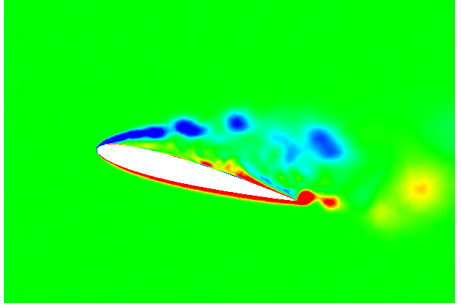

(a) Static

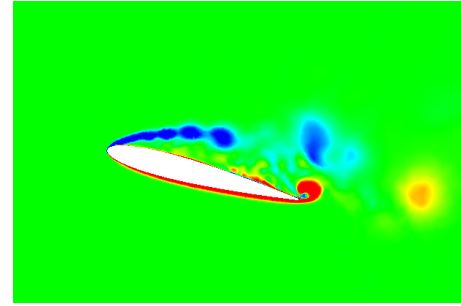

(b) Pitching, $k=0.025$

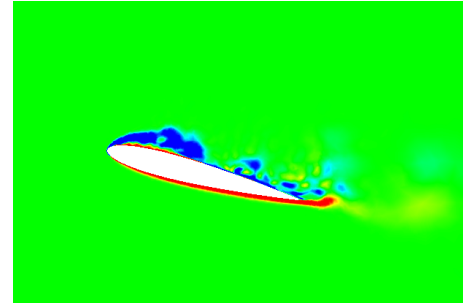

(c) Pitching, $k=0.1$

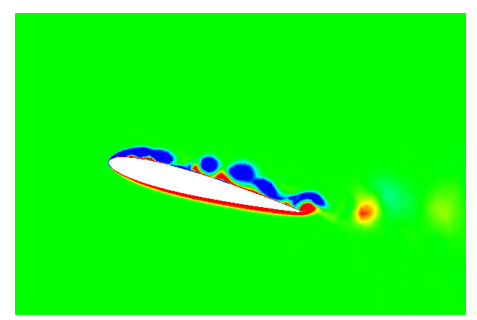

(d) Pitching, $k=0.2$
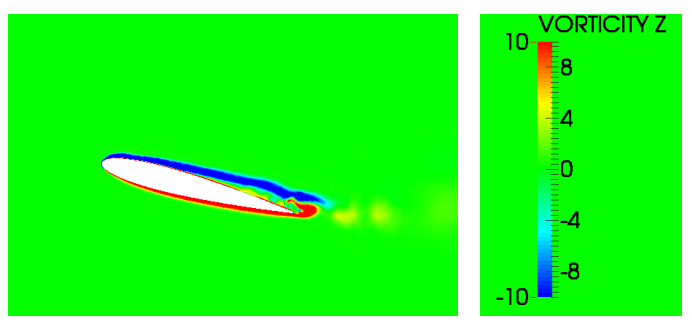

(e) Pitching, $k=0.4$

FIG. 9: Contours of non-dimensional spanwise vorticity, $\omega_{Z} c / V_{0}$, at airfoil midplane for a static airfoil and for airfoils pitching at various reduced frequencies. The angle of incidence is $\alpha=14.5^{\circ}$ (upstroke phase). 


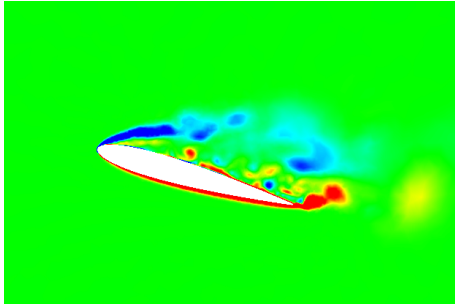

(a) Static

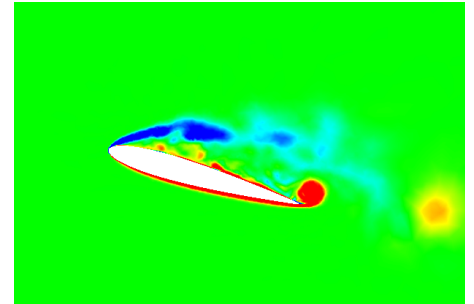

(b) Pitching, $k=0.025$

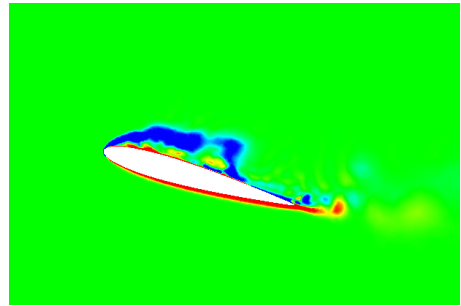

(c) Pitching, $k=0.1$

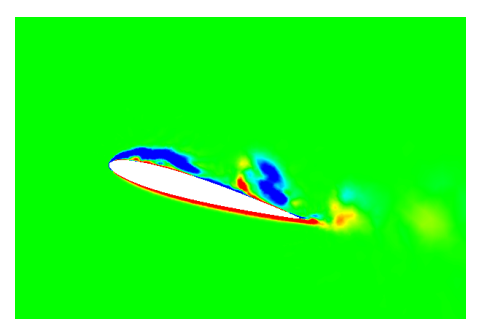

(d) Pitching, $k=0.2$

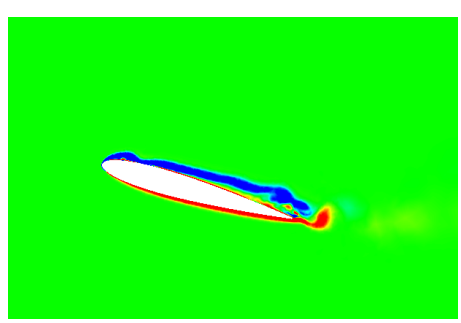

(e) Pitching, $k=0.4$

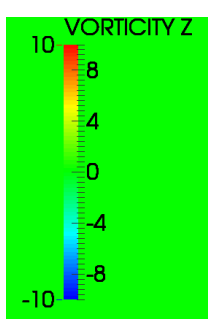$$
\sqrt{20}
$$

FIG. 10: Contours of non-dimensional spanwise vorticity, $\omega_{Z} c / V_{0}$, at airfoil midplane for a static airfoil and for airfoils pitching at various reduced frequencies. The angle of incidence is $\alpha=15.9^{\circ}$ (upstroke phase).

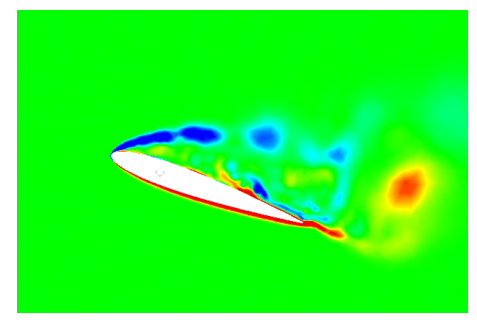

(a) Static

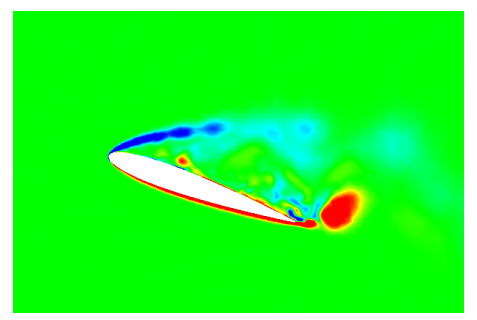

(b) Pitching, $k=0.025$

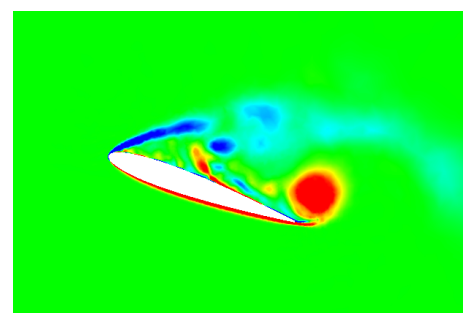

(c) Pitching, $k=0.1$

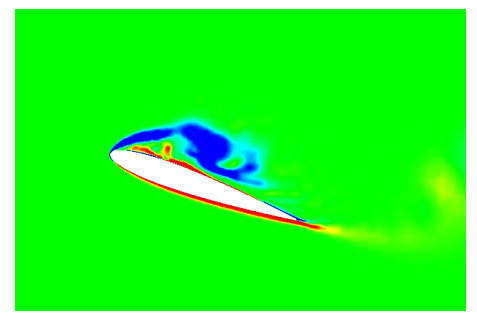

(d) Pitching, $k=0.2$

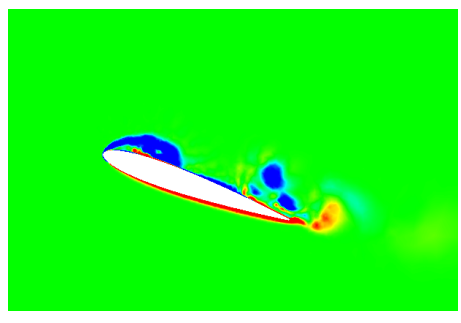

(e) Pitching, $k=0.4$

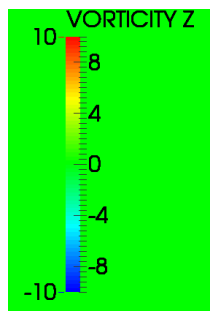

FIG. 11: Contours of non-dimensional spanwise vorticity, $\omega_{Z} c / V_{0}$, at airfoil midplane for a static airfoil and for airfoils pitching at various reduced frequencies. The angle of incidence is $\alpha=19.5^{\circ}$ (upstroke phase). 


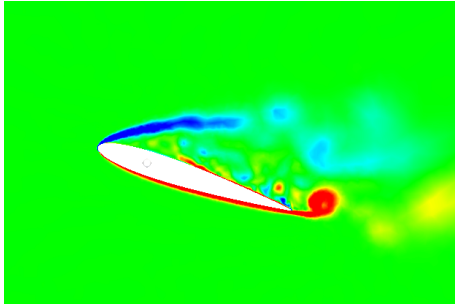

(a) Static

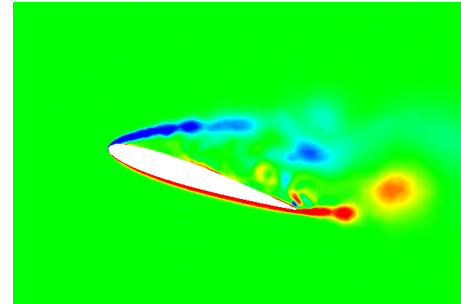

(b) Pitching, $k=0.025$

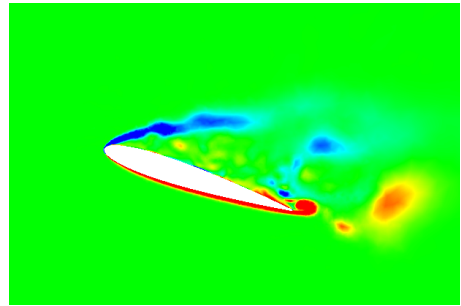

(c) Pitching, $k=0.1$

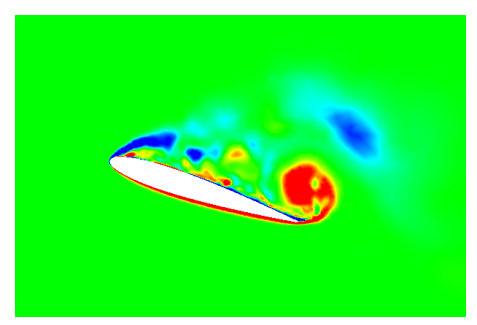

(d) Pitching, $k=0.2$
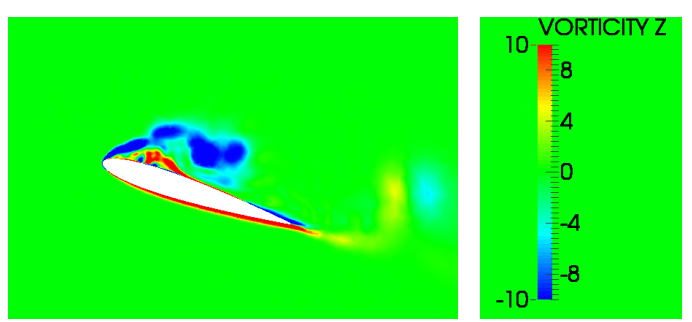

(e) Pitching, $k=0.4$

FIG. 12: Contours of non-dimensional spanwise vorticity, $\omega_{Z} c / V_{0}$, at airfoil midplane for a static airfoil and for airfoils pitching at various reduced frequencies. The angle of incidence is $\alpha=18.1^{\circ}$ (downstroke phase).

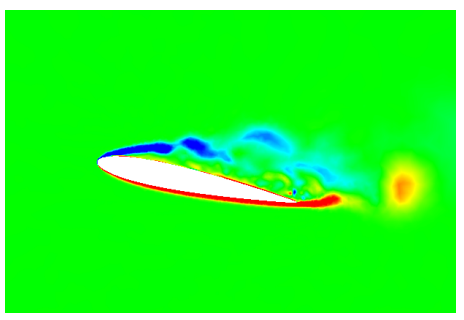

(a) Static

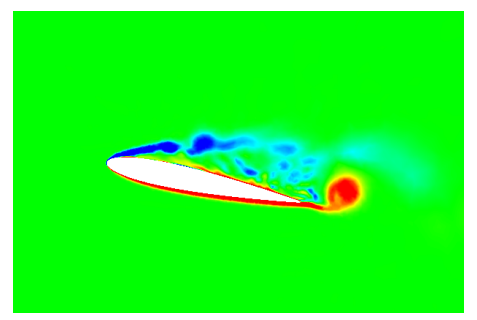

(b) Pitching, $k=0.025$

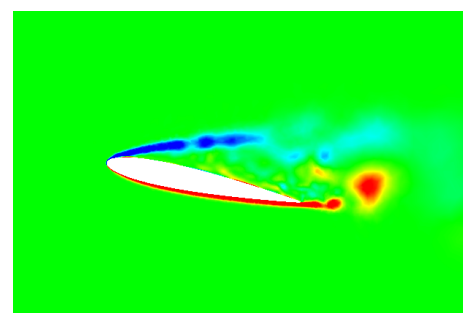

(c) Pitching, $k=0.1$

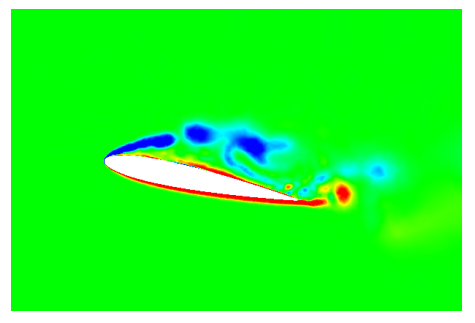

(d) Pitching, $k=0.2$

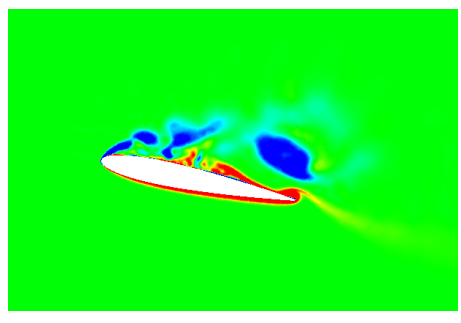

(e) Pitching, $k=0.4$

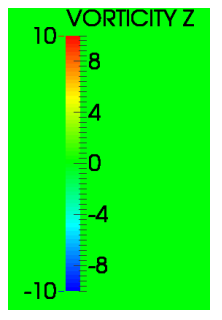

FIG. 13: Contours of non-dimensional spanwise vorticity, $\omega_{Z} c / V_{0}$, at airfoil midplane for a static airfoil and for airfoils pitching at various reduced frequencies. The angle of incidence is $\alpha=11.6^{\circ}$ (downstroke phase). 


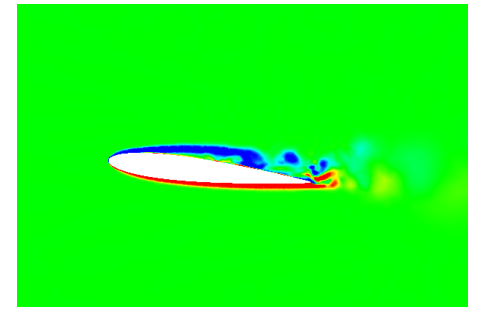

(a) Static

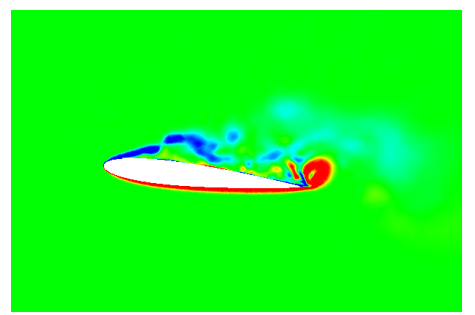

(d) Pitching, $k=0.2$

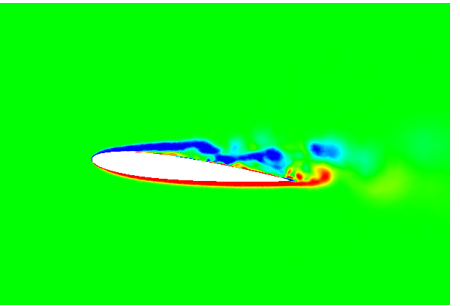

(b) Pitching, $k=0.025$

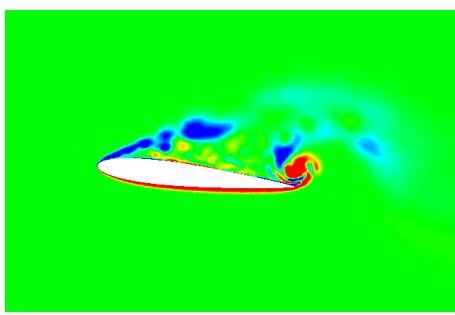

(e) Pitching, $k=0.4$

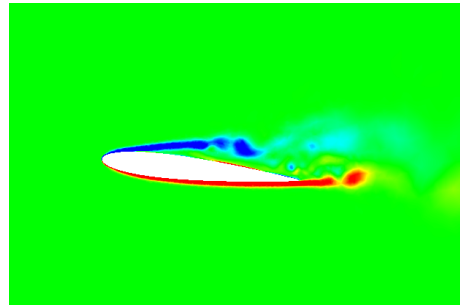

(c) Pitching, $k=0.1$

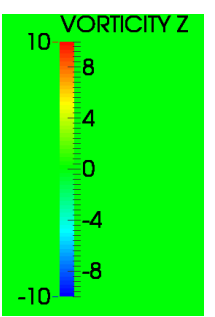

FIG. 14: Contours of non-dimensional spanwise vorticity, $\omega_{Z} c / V_{0}$, at airfoil midplane for a static airfoil and for airfoils pitching at various reduced frequencies. The angle of incidence is $\alpha=6.2^{\circ}$ (downstroke phase). 


\section{B. Boundary layer detachment}

The boundary layer detachment is investigated for the different pitching cases. Figure 15a shows the incidence for which $C_{f}$ local becomes negative during the upstroke phase as a function of the dimensionless position along the airfoil. It therefore represents the evolution of the boundary layer detachment point along the airfoil suction side. Positions $x / c=0$ and $x / c=1$ correspond to the leading edge and the trailing edge, respectively. $C_{f}$ local being negative during the entire cycle in the trailing edge vicinity, curves points do not reach $x / c=1$. The airfoil area where the boundary layer is always detached seems to be more important when the reduced frequency is high. The boundary layer detachment process goes through larger incidence ranges as the reduced frequency increases and the detachment point reaches the leading edge vicinity at higher incidences as the reduced frequency increases. Figure 15b shows the incidence for which the boundary layer detachment point reaches the leading edge vicinity as a function of the reduced frequency. A rise of the reduced frequency leads to an increase of the incidence for which the boundary layer detachment point reaches the leading edge vicinity but in a more important manner at low reduced frequencies than at high reduced frequencies.

After this part dedicated to the boundary layer detachment, the next section aims to investigate the Leading Edge Vortex.

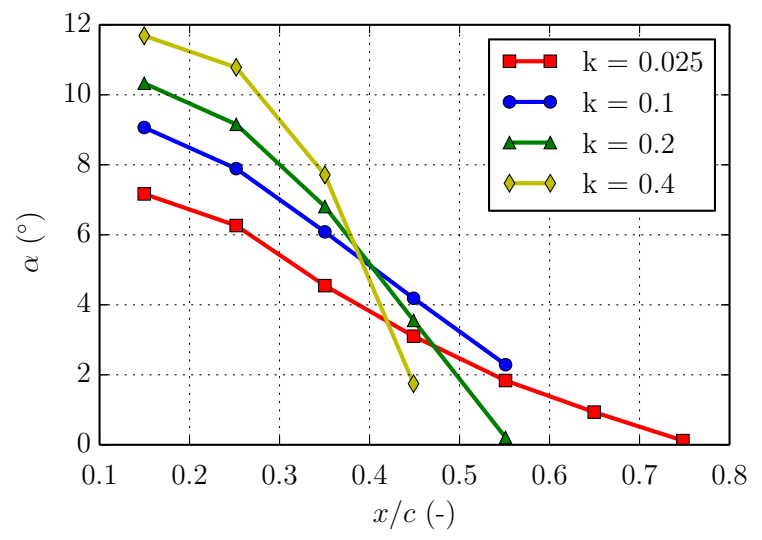

(a)

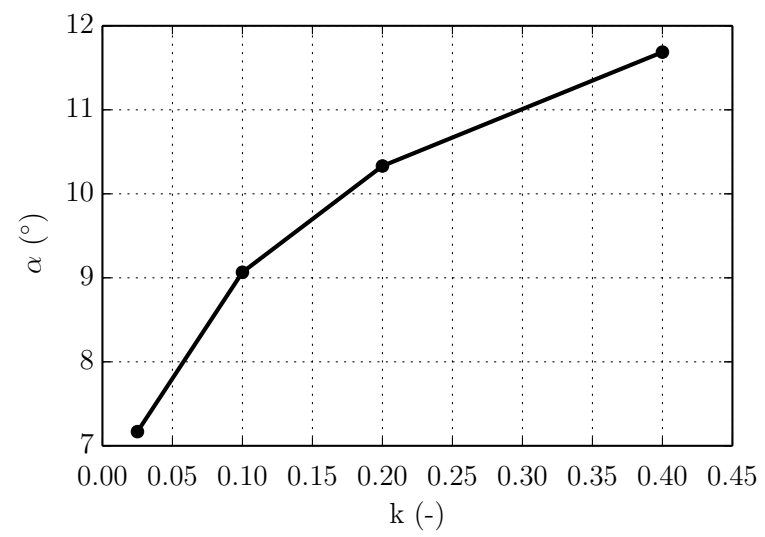

(b)

FIG. 15: (a) Position of the boundary layer detachment point as a function of the airfoil angle of incidence, (b) Angle of incidence for which the boundary layer detachment point reached the leading edge vicinity as a function of the reduced frequency. 


\section{Initiation, growth and shedding of the Leading Edge Vortex}
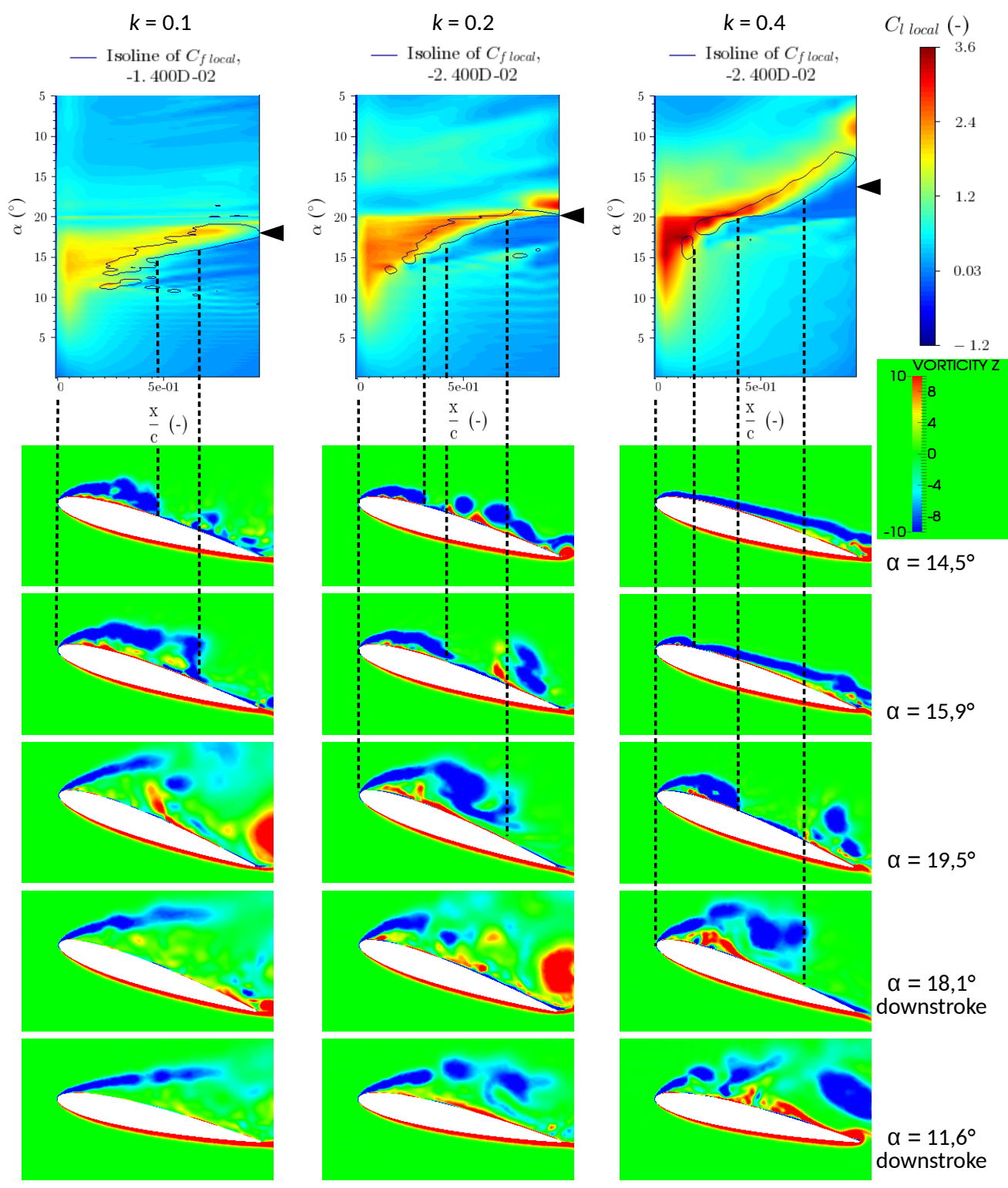

FIG. 16: Top: Local lift coefficient $C_{l}$ local as a function of the dimensionless position on the airfoil suction side and as a function of the incidence with an isovalue line of $C_{f}$ local ; Bottom: non-dimensional spanwise vorticity contours at airfoil midplane for several angles of incidence.

Pictures are scaled to observe the LEV influence on the local lift coefficient ; Left column: $k=0.1$; Central column: $k=0.2$; Right column: $k=0.4 ; \varangle$ : Angle of incidence at the beginning of the lift coefficient fall.

The present section focuses on the pitching cases where the LEV is clearly observed: $k=0.1$, $k=0.2$ and $k=0.4$. Top of figure 16 shows the local lift coefficient $C_{l}$ local as a function of 
the dimensionless position on the airfoil suction side and as a function of the incidence during one pitching cycle for each reduced frequency. These colormaps show the evolution of the lift distribution along the airfoil suction side during a pitching period. At the beginning of the upstroke phase, the lift is mostly generated at the leading edge vicinity. As the boundary layer detachment reaches the leading edge vicinity (at $\alpha \approx 9.1^{\circ}$ for $k=0.1, \alpha \approx 10.3^{\circ}$ for $k=0.2$ and $\alpha \approx 11.7^{\circ}$ for $k=0.4$ ), the high local lift coefficient area progressively extends along the airfoil suction side. The leading edge contribution to the lift then drops and a peak of local lift coefficient travels along the airfoil suction side from the leading edge to the trailing edge. Once this peak of local lift coefficient passed the trailing edge, the local lift coefficient is low over the whole airfoil suction side during the rest of the pitching period. The angle of incidence at the beginning of the lift coefficient fall has been reported by a black triangle on $C_{l \text { local }}$ colormaps. It happens at $\alpha \approx 18^{\circ}$ for $k=0.1, \alpha \approx 20^{\circ}$ for $k=0.2$ and $\alpha \approx 16.5^{\circ}$ in downstroke phase for $k=0.4$. The increase of the lift coefficient fall incidence delay with the rise of the reduced frequency is clearly visible on the $C_{l}$ local colormaps of figure 16.

The LEV is a strong vortex and generates a friction force oriented towards the airfoil leading edge. Isolines of $C_{f}$ local with negative values have been plotted on $C_{l}$ local colormaps of figure 16 to detect the LEV in the different pitching cases and their influence on $C_{l}$ local . The isolines highlight that the local lift coefficient peaks which travel along the airfoil suction side are generated by the LEV. As shown for example by Acharya et al. [4] and by Sharma and Poddar [34], a LEV generates a depression on the airfoil suction side, which leads to a local peak of the pressure coefficient and as a consequence of the local lift coefficient in the present study. As the LEV is generated at the leading edge vicinity, grows and convects along the airfoil until its shedding into the wake, the LEV generates a local lift coefficient peak that travels along the airfoil suction side. The depression generated by the LEV therefore increases the airfoil lift coefficient, but also increases the airfoil drag coefficient as shown by figure $6 \mathrm{~b}$.

Contours of non-dimensional spanwise vorticity at airfoil midplane are displayed below the $C_{\text {l local }}$ colormaps of figure 16 . The airfoil chord on the contours is scaled to the abscissa of $C_{l \text { local }}$ colormaps. Five angles of incidence are displayed and dashed lines report the LEV size on $C_{l \text { local }}$ colormaps. It shows that isolines of $C_{f}$ local detect only a part of the LEV. The flow over the airfoil suction side is complex. The principal vortex has a strong circulation and seems to generate counter-rotating vortices near the airfoil surface. This behaviour have been also observed experimentally by Mulleners and Raffel [7]. The friction forces produced by the LEV on the airfoil 
suction side is therefore not only negative.

Dashed lines of figure 16 report the LEV size on the $C_{\text {l local }}$ colormaps to analyse the LEV influence on the local lift coefficient distribution. The LEV leads to a high level of local lift coefficient on the surface it extends. For a given angle of incidence, the local lift coefficient maximum seems to be located under the LEV centre, where the depression generated by the LEV is the highest. The higher the reduced frequency, the higher the maximum local lift coefficient generated by the LEV. The LEV grows as the angle of incidence increases which extends the high local lift coefficient area on the airfoil suction side. The local lift coefficient maximum moves with the LEV centre. Then, the LEV gradually detaches from the leading edge, travels beyond the airfoil suction side before being shed into the wake, which leads to the displacement of the maximum of the local lift coefficient from the leading edge towards the trailing edge. The lift coefficient drops during the LEV shedding. Right after the shedding of the LEV into the wake, a surge of local lift coefficient is observed at the trailing edge vicinity. It seems to be due to a Trailing Edge Vortex (TEV) which is generated from the airfoil pressure side in response to the shedding of the LEV.

Following the boundary layer detachment, the LEV generation therefore leads to keep a high level of lift for a more or less important angular range and increases the incidence delay of the lift and drag coefficients drop.

This study was so far focussed on the LEV development as a function of the airfoil angle of incidence. The LEV seems to go through the same steps during its development independently to the reduced frequency but at higher angles of incidence as the reduced frequency increases. The rest of the study is focussed on the LEV development over time. The development time of the LEV will be evaluated with $\Delta t^{*}=\frac{\Delta t V_{0}}{c}$, the dimensionless time passed since the boundary layer detachment reached the leading edge vicinity. The colormaps on top of figure 17 show for each reduced frequency the local lift coefficient as a function of $\Delta t^{*}$ and as a function of the dimensionless position along the airfoil suction side. The incidence variation $\Delta \alpha$ corresponding to $\Delta t^{*}$ depends on the reduced frequency and is indicated on the right axis of the colormaps. Contours of non-dimensional spanwise vorticity for different $\Delta t^{*}$ have been reported below the colormaps for each reduced frequency. Corresponding incidence variations have been reported on each contour.

In the very first moment of the LEV generation, it seems to develop in a similar manner independently of the reduced frequency. The LEV occupies the same surface on the airfoil suction 
side, although the vortex is higher as the reduced frequency increases. The evolution of $C_{l}$ local over time is similar for the three pitching cases, although it differs on its amplitude.

Contours of non-dimensional spanwise vorticity of figure 17 show that at $\Delta t^{*}=3.1$, the pitching airfoil with $k=0.4$ is already in downstroke phase which seems to hurried the LEV detachment from the airfoil suction side. The LEV led to keep a high lift coefficient on an angular range $\Delta \alpha_{L E V}$ of slightly less than $12^{\circ}$. The pitching cases with $k=0.1$ and $k=0.2$ still are in upstroke phase and the LEV is still attached to the airfoil.

At $\Delta t^{*}=3.5$, in the pitching case with $k=0.2$, the evolution of local lift coefficient distribution over the airfoil suction side shows that the LEV gradually detaches. The airfoil is at the maximum angle of incidence. The LEV detachment therefore occurs when the airfoil rotation speed is close to zero and the angular interval crossed during the LEV detachment is small. The lift and drag coefficients as a function of incidence thus drop more brutally compared to the pitching case with $k=0.4$, as seen on figure 6 . In the pitching case with $k=0.2$, the LEV led to keep a high lift coefficient on an angular range $\Delta \alpha_{L E V}$ of approximately $9.7^{\circ}$.

It is only at $\Delta t^{*} \approx 5$ that the LEV of the pitching case with $k=0.1$ is shed into the wake. It occurs on an angular range where the airfoil rotation speed is higher than for the case $k=0.2$ but still small. The lift and drag coefficient drop as a function of incidence remains fairly brutal (cf. figure 6). Even though the LEV lifetime is the highest in the pitching case with $k=0.1$, the oscillation frequency being the lowest, the LEV generation led to keep a high lift coefficient on the smallest angular range, approximately equal to $8.8^{\circ}$.

In summary, in the very first moments of its generation, the LEV seems to develop over the time in a similar manner independently of the reduced frequency. The LEV is however more intense and generates higher maximum value of local lift coefficient as the reduced frequency increases. Its lifetime on the airfoil suction side then depends on the part of the oscillation cycle for which the LEV initiates. The shedding of the LEV into the wake seems to be hurried by the beginning of the airfoil downstroke phase. Therefore, the closer to the maximal angle of incidence the LEV initiates, the lower its lifetime on the airfoil suction side. Figure 18 shows the angular range $\Delta \alpha_{L E V}$ and the time range $\Delta t_{L E V}^{*}$ between the moment for which the boundary layer detachment reached the leading edge vicinity and the moment for which the lift and drag coefficients fall as a function of reduced frequency. An increase of the reduced frequency leading to a LEV initiation at higher angle of incidence, the LEV lifetime reduces as the reduced frequency increases. However, due to oscillation frequency differences, the angular range crossed during the LEV lifetime extends as the 
reduced frequency increases. The lift and drag coefficient drop incidence delay related to the LEV is therefore more important as the reduced frequency increases.

The pitching case with $k=0.2$ produces the highest maximum value of lift coefficient. In the pitching case with $k=0.1$, the LEV initiates early and is shed on the wake before the airfoil reached the maximal angle of incidence. In the pitching case with $k=0.4$, the LEV initiates late and is not fully developed when the airfoil reaches the maximal angle of incidence. It seems that in the reduced frequency range investigated it exists an optimal reduced frequency which leads to the highest maximal value of lift coefficient, corresponding to an optimal LEV generation.

\section{CONCLUSIONS}

In the present study, Large Eddy Simulations have been performed on a pitching NACA0012 airfoil. The aim of this study was to better understand the reduced frequency influence on the dynamic stall phenomenon. A local lift coefficient and a local quantity related to the friction on the airfoil surface have been introduced to analyse the flow influence on the airfoil suction side.

The stall of the flow around an airfoil is delayed in a pitching airfoil compared to a static case. This incidence delay has been divided into two main phases: a delay in the boundary layer detachment and the initiation and growth of a Leading Edge Vortex (LEV) which leads to keep a high level of lift.

A rise of the reduced frequency increases the incidence delay related to the boundary layer detachment. This increase of the incidence delay seems to be more pronounced if low reduced frequencies are considered.

The LEV initiates at higher angle of incidence as the reduced frequency increases. At its beginning, the LEV development over time does not seem to be influenced by the reduced frequency, even though a high reduced frequency leads to a more intense LEV and generates higher maximum value of local lift coefficient. However, the LEV lifetime over the airfoil suction side and consequently the time interval for which a high level of lift is kept could be shortened when the airfoil is close to the downstroke phase. The LEV lifetime and the lift and drag coefficient stall incidence delay therefore depends on the part of the oscillation cycle for which the LEV initiates. An increase of the reduced frequency leading to a LEV initiation at higher angle of incidence, the LEV lifetime reduces as the reduced frequency increases. However, as the oscillation frequency increases, the angular range crossed during the LEV lifetime extends as the reduced frequency increases. The lift and drag coefficient drop incidence delay related to the LEV is therefore more 
important as the reduced frequency increases. The incidence delay related to the LEV dominates the incidence delay related to the boundary layer detachment for all tested reduced frequencies.

\section{ACKNOWLEDGMENTS}

This work has been supported by the Hydrofluv Project, a Research Program piloted by Hydroquest. Vincent Moureau and Ghislain Lartigue from the CORIA lab, and the SUCCESS scientific group are acknowledged for providing the YALES2 code. Computations presented in this paper were performed using HPC resources from GENCI-IDRIS (Grant No. 2012-020611) and CIMENT infrastructure (supported by CPER07 13 CIRA and ANR-10-EQPX-29-01).

[1] W.J. McCroskey. The phenomenon of dynamic stall. Technical report, DTIC Document, 1981.

[2] L.W. Carr. Progress in analysis and prediction of dynamic stall. Journal of aircraft, 25(1):6-17, 1988.

[3] T.C. Corke and F.O. Thomas. Dynamic stall in pitching airfoils: aerodynamic damping and compressibility effects. Annual Review of Fluid Mechanics, 47:479-505, 2015.

[4] M. Acharya and M.H. Metwally. Unsteady pressure field and vorticity production over a pitching airfoil. AIAA journal, 30(2):403-411, 1992.

[5] T. Lee and P. Gerontakos. Investigation of flow over an oscillating airfoil. Journal of Fluid Mechanics, 512:313-341, 2004.

[6] K. Mulleners and M. Raffel. The onset of dynamic stall revisited. Experiments in fluids, 52(3):779$793,2012$.

[7] K. Mulleners and M. Raffel. Dynamic stall development. Experiments in fluids, 54(2):1469, 2013.

[8] D.E. Rival, J. Kriegseis, P. Schaub, A. Widmann, and C. Tropea. Characteristic length scales for vortex detachment on plunging profiles with varying leading-edge geometry. Experiments in fluids, $55(1): 1660,2014$.

[9] A. Widmann and C. Tropea. Parameters influencing vortex growth and detachment on unsteady aerodynamic profiles. Journal of Fluid Mechanics, 773:432-459, 2015.

[10] J.G. Leishman and T.S. Beddoes. A semi-empirical model for dynamic stall. Journal of the American Helicopter society, 34(3):3-17, 1989.

[11] S. Wang, D.B. Ingham, L. Ma, M. Pourkashanian, and Z. Tao. Numerical investigations on dynamic 
stall of low reynolds number flow around oscillating airfoils. Computers \& Fluids, 39(9):1529-1541, 2010.

[12] P. Wernert, W. Geissler, M. Raffel, and J. Kompenhans. Experimental and numerical investigations of dynamic stall on a pitching airfoil. AIAA journal, 34(5):982-989, 1996.

[13] G. Martinat, M. Braza, Y. Hoarau, and G. Harran. Turbulence modelling of the flow past a pitching naca0012 airfoil at 105 and 106 reynolds numbers. Journal of Fluids and Structures, 24(8):1294-1303, 2008.

[14] E. Berton, C. Allain, D. Favier, and C. Maresca. Experimental methods for subsonic flow measurements. Notes on numerical fluid mechanics and multidisciplinary design, 81:97-104, 2002.

[15] K.W. McAlister, L.W. Carr, and W.J. McCroskey. Dynamic stall experiments on the naca 0012 airfoil. Technical report, 1978.

[16] M. Sanchez-Rocha, M. Kirtas, and S. Menon. Zonal hybrid rans-les method for static and oscillating airfoils and wings. AIAA paper, 1256:2006, 2006.

[17] P.R. Spalart, S. Deck, M.L. Shur, K.D. Squires, M.K. Strelets, and A. Travin. A new version of detached-eddy simulation, resistant to ambiguous grid densities. Theoretical and computational fluid dynamics, 20(3):181-195, 2006.

[18] R.A. Piziali. 2-d and 3-d oscillating wing aerodynamics for a range of angles of attack including stall. Technical report, 1994.

[19] M. Rahromostaqim, A. Posa, and E. Balaras. Numerical investigation of the performance of pitching airfoils at high amplitudes. AIAA Journal, pages 2221-2232, 2016.

[20] C.S. Peskin. The immersed boundary method. Acta numerica, 11:479-517, 2002.

[21] A.W. Mackowski and C.H.K. Williamson. Direct measurement of thrust and efficiency of an airfoil undergoing pure pitching. Journal of Fluid Mechanics, 765:524-543, 2015.

[22] D. Garmann and M. Visbal. Implicit les computations for a rapidly pitching plate. AIAA Paper AIAA-2010-4282, AIAA, 2010.

[23] W.J. McCroskey, K.W. McAlister, L.W. Carr, S.L. Pucci, O. Lambert, and R.F. Indergrand. Dynamic stall on advanced airfoil sections. Journal of the American Helicopter Society, 26(3):40-50, 1981.

[24] L.W. Carr, K.W. McAlister, and W.J. McCroskey. Analysis of the development of dynamic stall based on oscillating airfoil experiments. Technical report, 1977.

[25] V. Moureau, P. Domingo, and L. Vervisch. Design of a massively parallel cfd code for complex 
geometries. Comptes Rendus Mécanique, 339(2):141-148, 2011.

[26] A.J. Chorin. Numerical solution of the navier-stokes equations. Mathematics of computation, 22(104):745-762, 1968.

[27] M. Malandain, N. Maheu, and V. Moureau. Optimization of the deflated conjugate gradient algorithm for the solving of elliptic equations on massively parallel machines. Journal of Computational Physics, 238:32-47, 2013.

[28] V. Moureau, P. Domingo, and L. Vervisch. From large-eddy simulation to direct numerical simulation of a lean premixed swirl flame: Filtered laminar flame-pdf modeling. Combustion and Flame, 158(7):1340-1357, 2011.

[29] L. Guedot, G. Lartigue, and V. Moureau. Design of implicit high-order filters on unstructured grids for the identification of large-scale features in large-eddy simulation and application to a swirl burner. Physics of Fluids (1994-present), 27(4):045107, 2015.

[30] N. Odier, G. Balarac, C. Corre, and V. Moureau. Numerical study of a flapping liquid sheet sheared by a high-speed stream. International Journal of Multiphase Flow, 77:196-208, 2015.

[31] M. Kraushaar. Application of the compressible and low-Mach number approaches to Large-Eddy Simulation of turbulent flows in aero-engines. PhD thesis, Institut National Polytechnique de ToulouseINPT, 2011.

[32] M. Germano, U. Piomelli, P. Moin, and W.H. Cabot. A dynamic subgrid-scale eddy viscosity model. Physics of Fluids A: Fluid Dynamics (1989-1993), 3(7):1760-1765, 1991.

[33] J.C.R. Hunt, A.A. Wray, and P. Moin. Eddies, streams, and convergence zones in turbulent flows. 1988.

[34] D.M. Sharma and K. Poddar. Investigation of dynamic stall characteristics for flow past an oscillating airfoil at various reduced frequencies by simultaneous piv and surface pressure measurements. In PIV13; 10th International Symposium on Particle Image Velocimetry, Delft, The Netherlands, July 1-3, 2013. Delft University of Technology, Faculty of Mechanical, Maritime and Materials Engineering, and Faculty of Aerospace Engineering, 2013. 

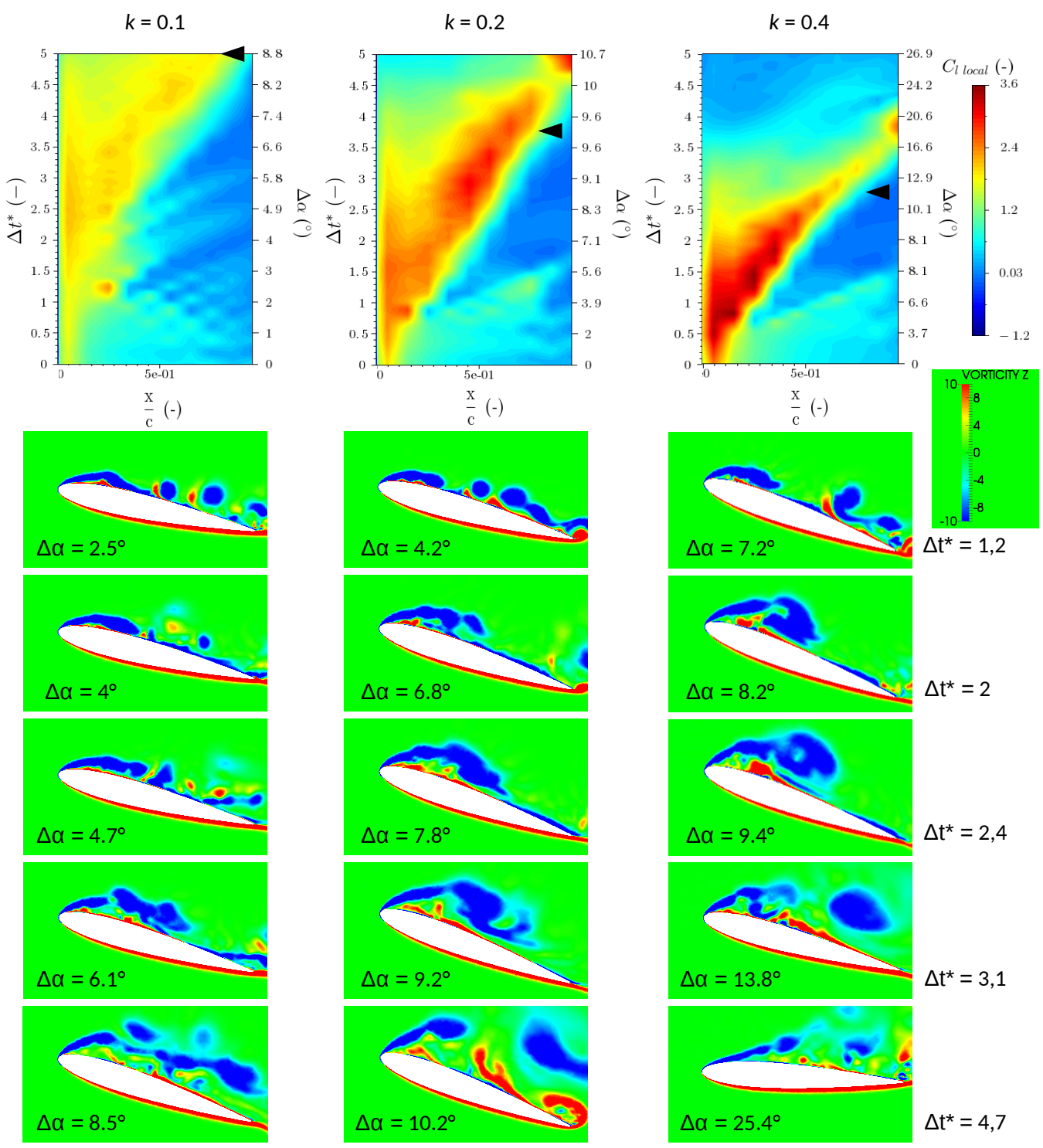

FIG. 17: Top: Local lift coefficient as a function of dimensionless position along the airfoil suction side and as a function of dimensionless passed time since the boundary layer detachment reaches the leading edge vicinity. Corresponding incidence variation is indicated on the right axis of each colormap ; Bottom: non-dimensional spanwise vorticity contours at airfoil midplane for several passed time since the boundary layer detachment reaches the leading edge vicinity. The corresponding incidence variation is indicated on each contour ; Left column: $k=0.1$; Central column: $k=0.2$; Right column: $k=0.4 ; \triangleleft$ : Angle of incidence at the beginning of the lift coefficient fall. 


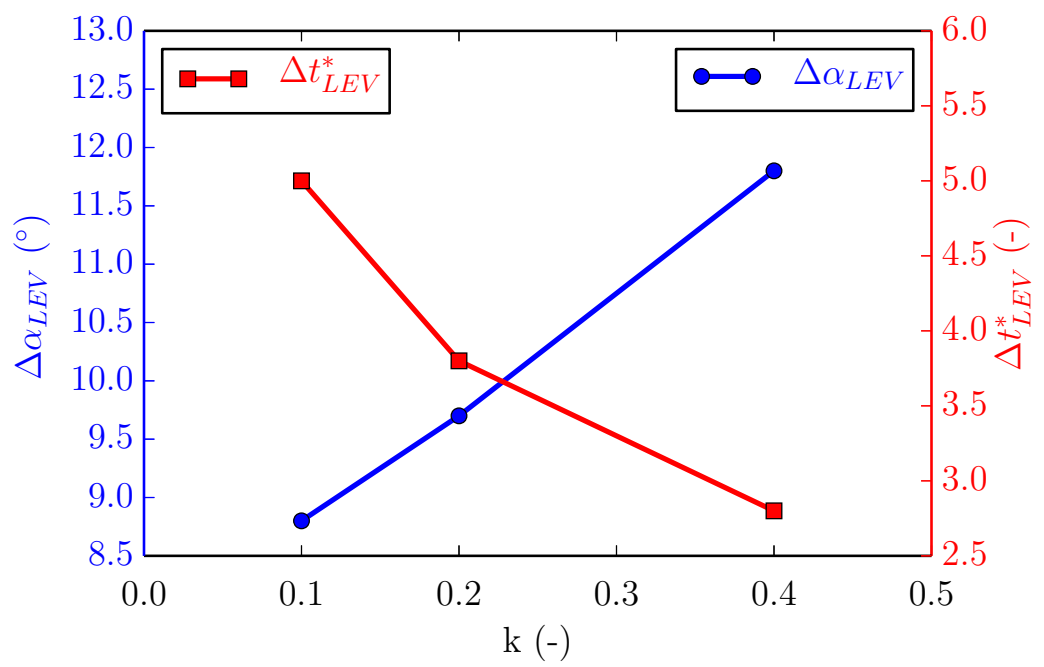

FIG. 18: Angular range $\Delta \alpha_{L E V}$ and dimensionless time range $\Delta t_{L E V}^{*}$ between the moment for which the boundary layer detachment reached the leading edge vicinity and the moment for which the lift and drag coefficients fall as a function of reduced frequency. 